Acta Crystallographica Section E

Structure Reports

Online

ISSN 1600-5368

\section{6'-Bromo-1'H-spiro[cyclohexane-1,2'- pyrido[2,3-d]pyrimidin $]-4^{\prime}\left(3^{\prime} H\right)$-one}

\section{Liupan Yang, Daxin Shi, Shu Chen, Hongxin Chai and Jiarong Li*}

School of Chemical Engineering and Environment, Beijing Institue of Technology, Beijing 100081, People's Republic of China

Correspondence e-mail: jrli@bit.edu.cn

Received 31 October 2011; accepted 4 December 2011

Key indicators: single-crystal X-ray study; $T=153 \mathrm{~K}$; mean $\sigma(\mathrm{C}-\mathrm{C})=0.003 \AA$; $R$ factor $=0.035 ; w R$ factor $=0.074 ;$ data-to-parameter ratio $=19.5$.

The title compound, $\mathrm{C}_{12} \mathrm{H}_{14} \mathrm{BrN}_{3} \mathrm{O}$, is built up from two fused six-membered rings and one six-membered ring linked through a spiro $\mathrm{C}$ atom. The hydropyrimidine ring has an envelope conformation and the cyclohexane ring is in a chair conformation. In the crystal, molecules are linked by $\mathrm{N}-$ $\mathrm{H} \cdots \mathrm{O}$ and $\mathrm{N}-\mathrm{H} \cdots \mathrm{N}$ hydrogen bonds, forming a molecular tape along the $b$ axis.

\section{Related literature}

For medicinal and biological properties of 2,3-dihydropyrido[2,3-d]-pyrimidin-4(1H)-one derivatives, see: Parish et al. (1982); Narayana et al. (2009). For related structures, see: Shi et al. (2010); Ling et al. (2009).

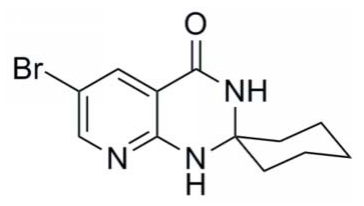

\section{Experimental}

\section{Crystal data}

$\mathrm{C}_{12} \mathrm{H}_{14} \mathrm{BrN}_{3} \mathrm{O}$

$M_{r}=296.17$

Monoclinic, $P 2_{1} / c$

$$
\begin{aligned}
& \beta=97.951(4)^{\circ} \\
& V=1181.7(6) \AA^{3} \\
& Z=4 \\
& \text { Mo } K \alpha \text { radiation }
\end{aligned}
$$

\section{Data collection}

Rigaku AFC10/Saturn724+ diffractometer

Absorption correction: multi-scan (CrystalClear; Rigaku/MSC 2009)

$$
T_{\min }=0.324, T_{\max }=0.732
$$

\section{Refinement}

$R\left[F^{2}>2 \sigma\left(F^{2}\right)\right]=0.035$

$w R\left(F^{2}\right)=0.074$

$S=1.00$

3160 reflections

162 parameters

Table 1

Hydrogen-bond geometry $\left(\AA{ }^{\circ}\right)$.

\begin{tabular}{lllll}
\hline$D-\mathrm{H} \cdots A$ & $D-\mathrm{H}$ & $\mathrm{H} \cdots A$ & $D \cdots A$ & $D-\mathrm{H} \cdots A$ \\
\hline $\mathrm{N} 1-\mathrm{H} 1 N \cdots \mathrm{N} 3^{\mathrm{i}}$ & $0.83(2)$ & $2.52(2)$ & $3.337(2)$ & $169(2)$ \\
$\mathrm{N} 2-\mathrm{H} 2 N \cdots \mathrm{O} 1^{\mathrm{ii}}$ & $0.83(2)$ & $1.98(2)$ & $2.807(2)$ & $175(2)$ \\
\hline
\end{tabular}

Symmetry codes: (i) $-x+1, y-\frac{1}{2},-z+\frac{1}{2}$; (ii) $-x+1, y+\frac{1}{2},-z+\frac{1}{2}$.

Data collection: CrystalClear (Rigaku/MSC, 2009); cell refinement: CrystalClear; data reduction: CrystalClear; program(s) used to solve structure: SHELXS97 (Sheldrick, 2008); program(s) used to refine structure: SHELXL97 (Sheldrick, 2008); molecular graphics: CrystalStructure (Rigaku/MSC, 2009); software used to prepare material for publication: CrystalStructure.

The authors thank Beijing Institute of Technology for the $\mathrm{X}$-ray diffraction analysis.

Supplementary data and figures for this paper are available from the IUCr electronic archives (Reference: IS5001).

\section{References}

Narayana, B., Rao, A. R. \& Rao, P. S. (2009). Eur. J. Med. Chem. 44, 1369 1376.

Ling, Z., Shi, D., Yanqiu, F., Wei, X. \& Li, J. (2009). Acta Cryst. E65, o1097.

Parish, H. A. Jr, Gilliom, R. D., Purcell, W. P., Browne, R. K., Spirk, R. F. \& White, H. D. (1982). J. Med. Chem. 25, 98-102.

Rigaku/MSC (2009). CrystalClear and CrystalStructure. Rigaku/MSC, The Woodlands, Texas, USA.

Sheldrick, G. M. (2008). Acta Cryst. A64, 112-122.

Shi, D., Yang, L., Tang, J., Wang, X. \& Li, J. (2010). Acta Cryst. E66, o2301. 


\section{supporting information}

Acta Cryst. (2012). E68, o178 [doi:10.1107/S1600536811052299]

\section{6'-Bromo-1'H-spiro[cyclohexane-1,2'-pyrido[2,3-d]pyrimidin]-4'(3'H)-one}

\section{Liupan Yang, Daxin Shi, Shu Chen, Hongxin Chai and Jiarong Li}

\section{S1. Comment}

2,3-Dihydropyrido[2,3-d]-pyrimidin-4(1H)-ones are a class of fused heterocycles which possess diuretic (Parish et al., 1982) and anti-bacterial activity (Narayana et al., 2009). 2-Substituted 2,3-dihydropyrido [2,3-d]pyrimidin-4(1H)-one derivatives can be obtained from the cyclocondensation of 2-amino-3-cyanopyridine with cyclopentanone (Shi et al., 2010). Here, we report the crystal structure of the title compound (Fig. 1). The molecular structure is built up with two fused six-membered ring and one six-membered ring linked through a spiro $\mathrm{C}$ atom. The pyrimidine ring has an envelope conformation, similar to that found in spiro \{cyclopentane- $1,2^{\prime}\left(1^{\prime} H\right)$ pyrido $\left[2^{\prime}, 3^{\prime}-d\right]$ pyrimidin- $4^{\prime}\left(3^{\prime} H\right)$-one (Shi et al., 2010). Cyclohexane ring has a similar chair conformation as cyclohexanespiro-2'-[2',3',6',7'-tetrahydro-1'H-cyclopenta[d]pyrimidin]-4'(5'H)-one (Ling et al., 2009). The crystal packing (Fig. 2) is stabilized by intermolecular $\mathrm{N}-\mathrm{H} \cdots \mathrm{O}$ and $\mathrm{N}-\mathrm{H} \cdots \mathrm{N}$ hydrogen bonds (Table 1 ).

\section{S2. Experimental}

A solution of 5-Br-2-amino-3-cyanopyridine $(2 \mathrm{mmol})$ and sodium methylate $(0.6 \mathrm{mmol})$ was refluxed in cyclohexanone ( $3 \mathrm{ml}$ ) for $10 \mathrm{~min}$. The reaction mixture was cooled to room temperature and then filtered to give the title compound. The product was recrystallizated from THF to give light yellow crystalline powder (m.p. 531-532 K). ${ }^{1} \mathrm{H}-\mathrm{NMR}$ (DMSO, p.p.m.):1.30-1.73 (10H, m, $\left.\mathrm{C}_{5} \mathrm{H}_{10}\right), 7.79(1 \mathrm{H}, \mathrm{s}, \mathrm{NH}), 7.92(1 \mathrm{H}, \mathrm{d}, \mathrm{J}=2.4 \mathrm{~Hz}$, Pyridine-H), $8.24(1 \mathrm{H}, \mathrm{d}, \mathrm{J}=2.4 \mathrm{~Hz}$, Pyridine-H), $8.35(1 H, \mathrm{~s}, \mathrm{NH})$; ESI-MS m/z: $[M+\mathrm{H}]^{+} 296.1 ; \mathrm{C}_{12} \mathrm{H}_{14} \mathrm{BrN}_{3} \mathrm{O}$ : calcd. C 48.67, H 4.76, N 14.19; found C 48.88, H 4.787, N 14.06 .

\section{S3. Refinement}

C-bounf $\mathrm{H}$ atoms were included in the riding model approximation, with $\mathrm{C}-\mathrm{H}=0.95-0.99 \AA$, and with $U_{\text {iso }}(\mathrm{H})=$ $1.2 U_{\mathrm{eq}}(\mathrm{C})$ or $1.5 U_{\mathrm{eq}}$ (methyl C), while the $\mathrm{N}-$ bound $\mathrm{H}$ atoms were refined freely $[\mathrm{N}-\mathrm{H}=0.83$ (2) $\AA]$. 


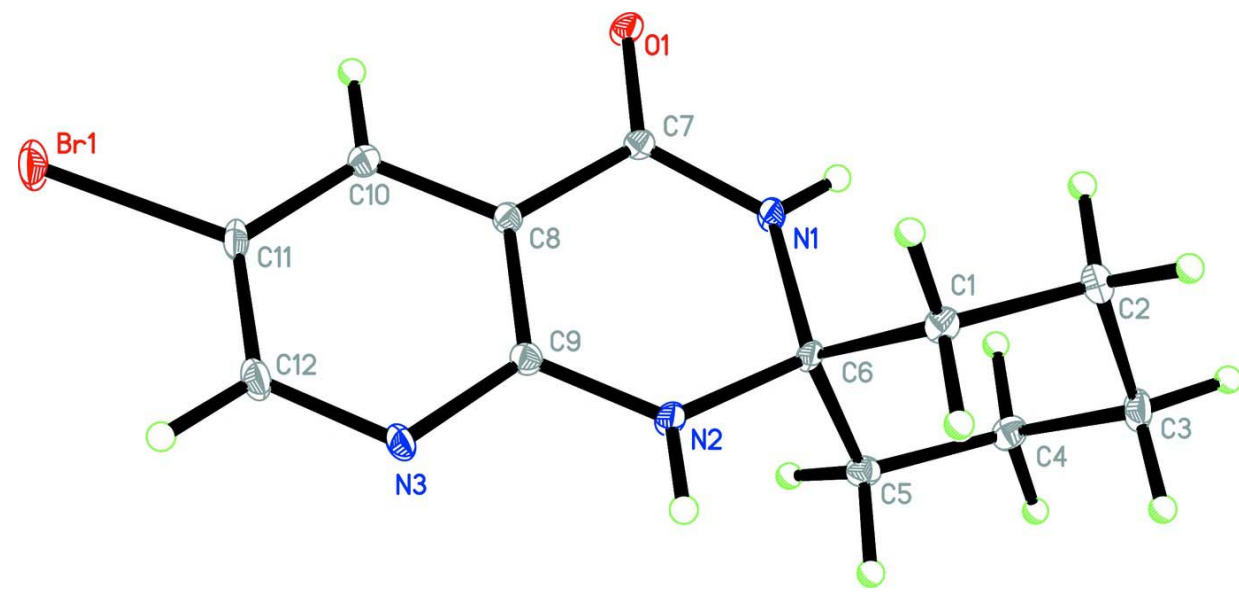

\section{Figure 1}

The molecular structure of the title compound with the atom numbering scheme. Displacement ellipsoids are drawn at the $30 \%$ probability level.

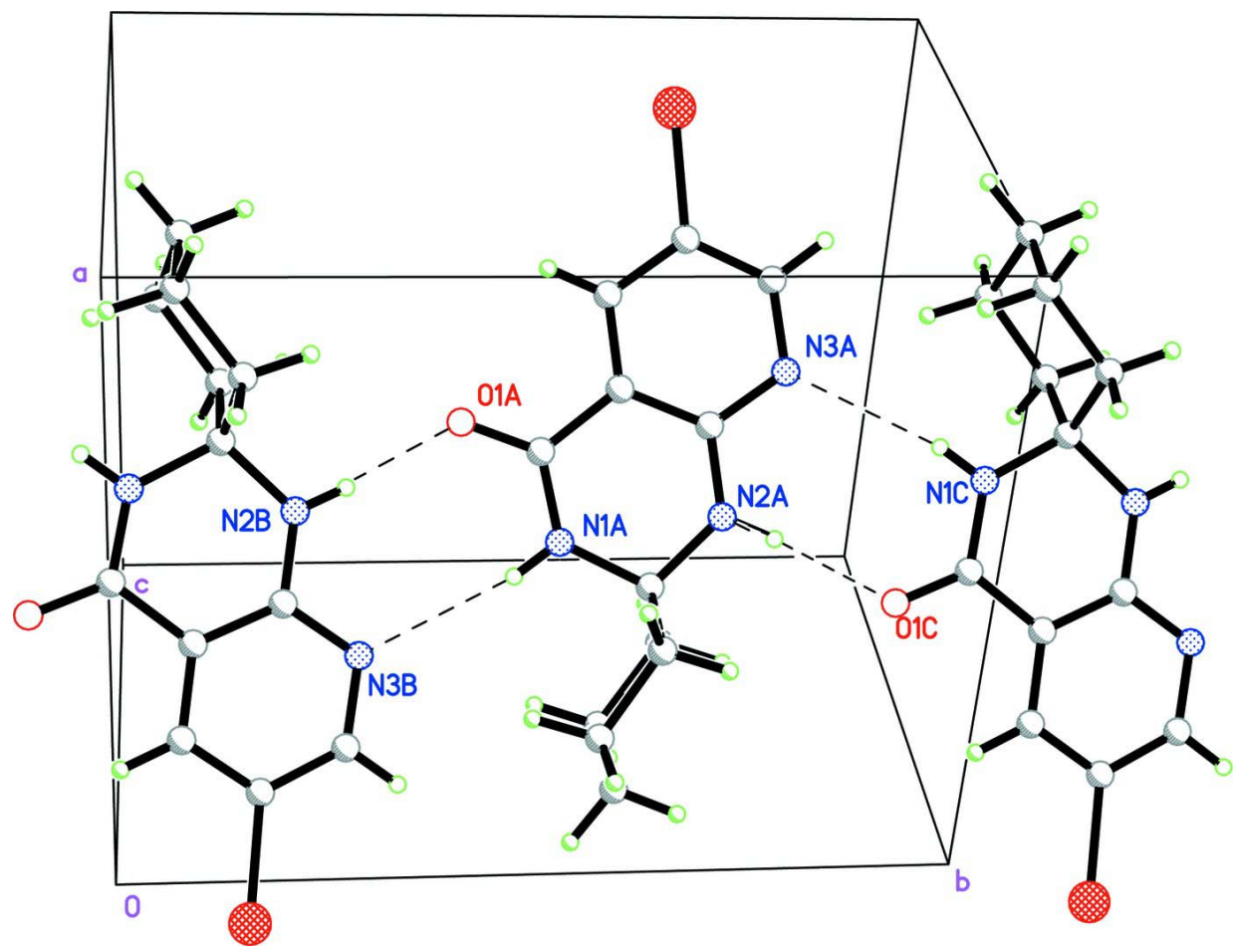

Figure 2

A packing diagram, showing $\mathrm{N}-\mathrm{H} \cdots \mathrm{O}$ and $\mathrm{N}-\mathrm{H} \cdots \mathrm{N}$ interactions (dotted lines) in the crystal structure of the title compound.

6'-Bromo-1'H-spiro[cyclohexane-1, 2'-pyrido[2,3- d] pyrimidin]-4' $\left(3^{\prime} H\right)$-one

Crystal data

$\mathrm{C}_{12} \mathrm{H}_{14} \mathrm{BrN}_{3} \mathrm{O}$

$M_{r}=296.17$

Monoclinic, $P 2_{1} / c$

Hall symbol: -P 2ybc

$a=10.591$ (3) $\AA$

$b=12.359$ (3) $\AA$ 
$c=9.116(3) \AA$

$\beta=97.951(4)^{\circ}$

$V=1181.7(6) \AA^{3}$

$Z=4$

$F(000)=600$

$D_{\mathrm{x}}=1.665 \mathrm{Mg} \mathrm{m}^{-3}$

Mo $K \alpha$ radiation, $\lambda=0.71073 \AA$

Data collection

Rigaku AFC10/Saturn724+

diffractometer

Radiation source: Rotating Anode

Graphite monochromator

Detector resolution: 28.5714 pixels $\mathrm{mm}^{-1}$

$\varphi$ and $\omega$ scans

Absorption correction: multi-scan

(CrystalClear; Rigaku/MSC, 2009)

$T_{\min }=0.324, T_{\max }=0.732$

Refinement

Refinement on $F^{2}$

Least-squares matrix: full

$R\left[F^{2}>2 \sigma\left(F^{2}\right)\right]=0.035$

$w R\left(F^{2}\right)=0.074$

$S=1.00$

3160 reflections

162 parameters

0 restraints

Primary atom site location: structure-invariant direct methods
Cell parameters from 3621 reflections

$\theta=2.6-29.1^{\circ}$

$\mu=3.47 \mathrm{~mm}^{-1}$

$T=153 \mathrm{~K}$

Platelet, colourless

$0.40 \times 0.24 \times 0.09 \mathrm{~mm}$

10128 measured reflections

3160 independent reflections

2283 reflections with $I>2 \sigma(I)$

$R_{\text {int }}=0.046$

$\theta_{\max }=29.1^{\circ}, \theta_{\min }=2.6^{\circ}$

$h=-14 \rightarrow 12$

$k=-16 \rightarrow 16$

$l=-12 \rightarrow 12$
Secondary atom site location: difference Fourier map

Hydrogen site location: inferred from neighbouring sites

$\mathrm{H}$ atoms treated by a mixture of independent and constrained refinement

$w=1 /\left[\sigma^{2}\left(F_{\mathrm{o}}{ }^{2}\right)+(0.0295 P)^{2}+0.126 P\right]$ where $P=\left(F_{\mathrm{o}}^{2}+2 F_{\mathrm{c}}^{2}\right) / 3$

$(\Delta / \sigma)_{\max }=0.001$

$\Delta \rho_{\max }=0.55$ e $\AA^{-3}$

$\Delta \rho_{\min }=-0.66 \mathrm{e} \AA^{-3}$

\section{Special details}

Experimental. Spectral data: IR (KBr): 3274, 3175, 2927, 1677, 1610, $1422 \mathrm{~cm}^{-1}$.

Geometry. All e.s.d.'s (except the e.s.d. in the dihedral angle between two 1.s. planes) are estimated using the full covariance matrix. The cell e.s.d.'s are taken into account individually in the estimation of e.s.d.'s in distances, angles and torsion angles; correlations between e.s.d.'s in cell parameters are only used when they are defined by crystal symmetry. An approximate (isotropic) treatment of cell e.s.d.'s is used for estimating e.s.d.'s involving 1.s. planes.

Refinement. Refinement of $F^{2}$ against ALL reflections. The weighted $R$-factor $w R$ and goodness of fit $S$ are based on $F^{2}$, conventional $R$-factors $R$ are based on $F$, with $F$ set to zero for negative $F^{2}$. The threshold expression of $F^{2}>\sigma\left(F^{2}\right)$ is used only for calculating $R$-factors(gt) etc. and is not relevant to the choice of reflections for refinement. $R$-factors based on $F^{2}$ are statistically about twice as large as those based on $F$, and $R$ - factors based on ALL data will be even larger.

Fractional atomic coordinates and isotropic or equivalent isotropic displacement parameters $\left(\AA^{2}\right)$

\begin{tabular}{lllll}
\hline & $x$ & $y$ & $z$ & $U_{\text {iso }} * / U_{\text {eq }}$ \\
\hline Br1 & $0.99820(2)$ & $0.65490(2)$ & $0.61599(3)$ & $0.03640(10)$ \\
O1 & $0.58435(14)$ & $0.40734(11)$ & $0.39085(16)$ & $0.0171(3)$ \\
N1 & $0.45035(17)$ & $0.52063(14)$ & $0.2500(2)$ & $0.0151(4)$ \\
N2 & $0.49356(17)$ & $0.71034(14)$ & $0.2458(2)$ & $0.0188(4)$ \\
N3 & $0.68151(16)$ & $0.78374(13)$ & $0.3597(2)$ & $0.0176(4)$ \\
C1 & $0.2735(2)$ & $0.64979(16)$ & $0.2411(2)$ & $0.0150(4)$ \\
H1A & 0.2814 & 0.6418 & 0.3501 & $0.018^{*}$
\end{tabular}




$\begin{array}{lllll}\text { H1B } & 0.2496 & 0.7257 & 0.2164 & 0.018^{*} \\ \text { C2 } & 0.1676(2) & 0.57477(17) & 0.1687(2) & 0.0179(5) \\ \text { H2A } & 0.1865 & 0.4994 & 0.2015 & 0.021^{*} \\ \text { H2B } & 0.0855 & 0.5960 & 0.2005 & 0.021^{*} \\ \text { C3 } & 0.1565(2) & 0.58081(18) & -0.0002(2) & 0.0194(5) \\ \text { H3A } & 0.0902 & 0.5296 & -0.0448 & 0.023^{*} \\ \text { H3B } & 0.1303 & 0.6547 & -0.0336 & 0.023^{*} \\ \text { C4 } & 0.2831(2) & 0.55308(17) & -0.0524(2) & 0.0172(5) \\ \text { H4A } & 0.2751 & 0.5617 & -0.1613 & 0.021^{*} \\ \text { H4B } & 0.3046 & 0.4765 & -0.0285 & 0.021^{*} \\ \text { C5 } & 0.3902(2) & 0.62565(16) & 0.0209(2) & 0.0160(4) \\ \text { H5A } & 0.3743 & 0.7006 & -0.0152 & 0.019^{*} \\ \text { H5B } & 0.4717 & 0.6014 & -0.0098 & 0.019^{*} \\ \text { C6 } & 0.40340(19) & 0.62564(15) & 0.1905(2) & 0.0129(4) \\ \text { C7 } & 0.55747(19) & 0.49967(15) & 0.3433(2) & 0.0127(4) \\ \text { C8 } & 0.64262(19) & 0.59204(16) & 0.3832(2) & 0.0125(4) \\ \text { C9 } & 0.6063(2) & 0.69577(16) & 0.3293(2) & 0.0139(4) \\ \text { C10 } & 0.7594(2) & 0.57798(17) & 0.4683(2) & 0.0174(5) \\ \text { H10 } & 0.7863 & 0.5086 & 0.5051 & 0.021^{*} \\ \text { C11 } & 0.8365(2) & 0.66793(17) & 0.4987(3) & 0.0203(5) \\ \text { C12 } & 0.7942(2) & 0.76778(17) & 0.4435(3) & 0.0203(5) \\ \text { H12 } & 0.8482 & 0.8286 & 0.4665 & 0.024^{*} \\ \text { H1N } & 0.408(2) & 0.4668(17) & 0.220(2) & 0.015(6)^{*} \\ \text { H2N } & 0.474(2) & 0.7700(19) & 0.208(3) & 0.026(7)^{*} \\ \end{array}$

Atomic displacement parameters $\left(\AA^{2}\right)$

\begin{tabular}{lllllll}
\hline & $U^{11}$ & $U^{22}$ & $U^{33}$ & $U^{12}$ & $U^{13}$ & $U^{23}$ \\
\hline Br1 & $0.02057(13)$ & $0.02781(14)$ & $0.05373(19)$ & $-0.00860(11)$ & $-0.01997(11)$ & $0.01191(13)$ \\
O1 & $0.0199(8)$ & $0.0096(7)$ & $0.0194(8)$ & $0.0000(6)$ & $-0.0054(6)$ & $0.0021(6)$ \\
N1 & $0.0143(9)$ & $0.0080(8)$ & $0.0209(10)$ & $-0.0027(7)$ & $-0.0049(7)$ & $-0.0002(7)$ \\
N2 & $0.0163(10)$ & $0.0070(9)$ & $0.0298(11)$ & $-0.0006(7)$ & $-0.0085(8)$ & $0.0047(8)$ \\
N3 & $0.0143(10)$ & $0.0111(9)$ & $0.0259(11)$ & $-0.0045(7)$ & $-0.0027(8)$ & $0.0016(8)$ \\
C1 & $0.0165(11)$ & $0.0128(10)$ & $0.0153(10)$ & $0.0017(9)$ & $0.0011(8)$ & $-0.0012(8)$ \\
C2 & $0.0133(11)$ & $0.0189(11)$ & $0.0216(12)$ & $-0.0025(9)$ & $0.0031(9)$ & $-0.0022(9)$ \\
C3 & $0.0130(11)$ & $0.0208(11)$ & $0.0227(12)$ & $-0.0036(9)$ & $-0.0029(9)$ & $-0.0027(10)$ \\
C4 & $0.0194(11)$ & $0.0184(10)$ & $0.0131(10)$ & $0.0000(9)$ & $-0.0003(8)$ & $-0.0017(9)$ \\
C5 & $0.0167(11)$ & $0.0145(10)$ & $0.0175(11)$ & $0.0032(8)$ & $0.0050(9)$ & $0.0026(9)$ \\
C6 & $0.0117(10)$ & $0.0070(9)$ & $0.0182(11)$ & $0.0004(8)$ & $-0.0042(8)$ & $0.0004(8)$ \\
C7 & $0.0135(10)$ & $0.0112(10)$ & $0.0132(10)$ & $0.0009(8)$ & $0.0013(8)$ & $-0.0005(8)$ \\
C8 & $0.0129(10)$ & $0.0113(10)$ & $0.0130(10)$ & $-0.0002(8)$ & $0.0006(8)$ & $-0.0005(8)$ \\
C9 & $0.0137(10)$ & $0.0111(9)$ & $0.0165(11)$ & $-0.0006(8)$ & $0.0010(8)$ & $-0.0003(8)$ \\
C10 & $0.0153(11)$ & $0.0151(10)$ & $0.0204(11)$ & $-0.0008(9)$ & $-0.0020(9)$ & $0.0041(9)$ \\
C11 & $0.0120(10)$ & $0.0200(11)$ & $0.0262(12)$ & $-0.0040(9)$ & $-0.0064(9)$ & $0.0027(10)$ \\
C12 & $0.0161(11)$ & $0.0148(10)$ & $0.0281(13)$ & $-0.0052(9)$ & $-0.0034(9)$ & $-0.0019(10)$ \\
& & & & & & \\
\hline
\end{tabular}


Geometric parameters $\left(\AA,{ }^{\circ}\right)$

\begin{tabular}{|c|c|c|c|}
\hline $\mathrm{Br} 1-\mathrm{C} 11$ & $1.896(2)$ & $\mathrm{C} 3-\mathrm{C} 4$ & $1.523(3)$ \\
\hline $\mathrm{O} 1-\mathrm{C} 7$ & $1.240(2)$ & $\mathrm{C} 3-\mathrm{H} 3 \mathrm{~A}$ & 0.9900 \\
\hline $\mathrm{N} 1-\mathrm{C} 7$ & $1.346(2)$ & $\mathrm{C} 3-\mathrm{H} 3 \mathrm{~B}$ & 0.9900 \\
\hline $\mathrm{N} 1-\mathrm{C} 6$ & $1.467(2)$ & $\mathrm{C} 4-\mathrm{C} 5$ & 1.525 \\
\hline $\mathrm{N} 1-\mathrm{H} 1 \mathrm{~N}$ & $0.83(2)$ & $\mathrm{C} 4-\mathrm{H} 4 \mathrm{~A}$ & 0.9900 \\
\hline $\mathrm{N} 2-\mathrm{C} 9$ & $1.336(3)$ & $\mathrm{C} 4-\mathrm{H} 4 \mathrm{~B}$ & 0.9900 \\
\hline $\mathrm{N} 2-\mathrm{C} 6$ & $1.459(3)$ & $\mathrm{C} 5-\mathrm{C} 6$ & $1.533(3)$ \\
\hline $\mathrm{N} 2-\mathrm{H} 2 \mathrm{~N}$ & $0.83(2)$ & $\mathrm{C} 5-\mathrm{H} 5 \mathrm{~A}$ & 0.9900 \\
\hline N3-C12 & $1.339(3)$ & $\mathrm{C} 5-\mathrm{H} 5 \mathrm{~B}$ & 0.9900 \\
\hline $\mathrm{N} 3-\mathrm{C} 9$ & $1.354(3)$ & $\mathrm{C} 7-\mathrm{C} 8$ & $1.469(3)$ \\
\hline $\mathrm{C} 1-\mathrm{C} 2$ & $1.533(3)$ & $\mathrm{C} 8-\mathrm{C} 10$ & $1.377(3)$ \\
\hline $\mathrm{C} 1-\mathrm{C} 6$ & $1.539(3)$ & $\mathrm{C} 8-\mathrm{C} 9$ & $1.407(3)$ \\
\hline $\mathrm{C} 1-\mathrm{H} 1 \mathrm{~A}$ & 0.9900 & $\mathrm{C} 10-\mathrm{C} 11$ & $1.384(3)$ \\
\hline $\mathrm{C} 1-\mathrm{H} 1 \mathrm{~B}$ & 0.9900 & $\mathrm{C} 10-\mathrm{H} 10$ & 0.9500 \\
\hline $\mathrm{C} 2-\mathrm{C} 3$ & $1.530(3)$ & $\mathrm{C} 11-\mathrm{C} 12$ & $1.384(3)$ \\
\hline $\mathrm{C} 2-\mathrm{H} 2 \mathrm{~A}$ & 0.9900 & $\mathrm{C} 12-\mathrm{H} 12$ & 0.9500 \\
\hline $\mathrm{C} 2-\mathrm{H} 2 \mathrm{~B}$ & 0.9900 & & \\
\hline $\mathrm{C} 7-\mathrm{N} 1-\mathrm{C} 6$ & $128.11(17)$ & $\mathrm{C} 4-\mathrm{C} 5-\mathrm{C} 6$ & $113.60(18)$ \\
\hline $\mathrm{C} 7-\mathrm{N} 1-\mathrm{H} 1 \mathrm{~N}$ & $115.1(15)$ & $\mathrm{C} 4-\mathrm{C} 5-\mathrm{H} 5 \mathrm{~A}$ & 108.8 \\
\hline $\mathrm{C} 6-\mathrm{N} 1-\mathrm{H} 1 \mathrm{~N}$ & $116.7(15)$ & $\mathrm{C} 6-\mathrm{C} 5-\mathrm{H} 5 \mathrm{~A}$ & 108.8 \\
\hline $\mathrm{C} 9-\mathrm{N} 2-\mathrm{C} 6$ & $126.21(17)$ & $\mathrm{C} 4-\mathrm{C} 5-\mathrm{H} 5 \mathrm{~B}$ & 108.8 \\
\hline $\mathrm{C} 9-\mathrm{N} 2-\mathrm{H} 2 \mathrm{~N}$ & $120.3(17)$ & $\mathrm{C} 6-\mathrm{C} 5-\mathrm{H} 5 \mathrm{~B}$ & 108.8 \\
\hline $\mathrm{C} 6-\mathrm{N} 2-\mathrm{H} 2 \mathrm{~N}$ & $112.6(17)$ & $\mathrm{H} 5 \mathrm{~A}-\mathrm{C} 5-\mathrm{H} 5 \mathrm{~B}$ & 107.7 \\
\hline $\mathrm{C} 12-\mathrm{N} 3-\mathrm{C} 9$ & $116.82(17)$ & $\mathrm{N} 2-\mathrm{C} 6-\mathrm{N} 1$ & $109.55(16)$ \\
\hline $\mathrm{C} 2-\mathrm{C} 1-\mathrm{C} 6$ & $112.62(16)$ & $\mathrm{N} 2-\mathrm{C} 6-\mathrm{C} 5$ & $108.24(17)$ \\
\hline $\mathrm{C} 2-\mathrm{C} 1-\mathrm{H} 1 \mathrm{~A}$ & 109.1 & $\mathrm{~N} 1-\mathrm{C} 6-\mathrm{C} 5$ & $110.62(17)$ \\
\hline $\mathrm{C} 6-\mathrm{C} 1-\mathrm{H} 1 \mathrm{~A}$ & 109.1 & $\mathrm{~N} 2-\mathrm{C} 6-\mathrm{C} 1$ & $109.04(16)$ \\
\hline $\mathrm{C} 2-\mathrm{C} 1-\mathrm{H} 1 \mathrm{~B}$ & 109.1 & $\mathrm{~N} 1-\mathrm{C} 6-\mathrm{C} 1$ & $109.38(17)$ \\
\hline $\mathrm{C} 6-\mathrm{C} 1-\mathrm{H} 1 \mathrm{~B}$ & 109.1 & $\mathrm{C} 5-\mathrm{C} 6-\mathrm{C} 1$ & $109.98(16)$ \\
\hline $\mathrm{H} 1 \mathrm{~A}-\mathrm{C} 1-\mathrm{H} 1 \mathrm{~B}$ & 107.8 & $\mathrm{O} 1-\mathrm{C} 7-\mathrm{N} 1$ & $122.07(18)$ \\
\hline $\mathrm{C} 3-\mathrm{C} 2-\mathrm{C} 1$ & $110.73(17)$ & $\mathrm{O} 1-\mathrm{C} 7-\mathrm{C} 8$ & $121.69(18)$ \\
\hline $\mathrm{C} 3-\mathrm{C} 2-\mathrm{H} 2 \mathrm{~A}$ & 109.5 & $\mathrm{~N} 1-\mathrm{C} 7-\mathrm{C} 8$ & $116.22(17)$ \\
\hline $\mathrm{C} 1-\mathrm{C} 2-\mathrm{H} 2 \mathrm{~A}$ & 109.5 & $\mathrm{C} 10-\mathrm{C} 8-\mathrm{C} 9$ & $119.49(18)$ \\
\hline $\mathrm{C} 3-\mathrm{C} 2-\mathrm{H} 2 \mathrm{~B}$ & 109.5 & $\mathrm{C} 10-\mathrm{C} 8-\mathrm{C} 7$ & $120.94(18)$ \\
\hline $\mathrm{C} 1-\mathrm{C} 2-\mathrm{H} 2 \mathrm{~B}$ & 109.5 & $\mathrm{C} 9-\mathrm{C} 8-\mathrm{C} 7$ & $119.53(18)$ \\
\hline $\mathrm{H} 2 \mathrm{~A}-\mathrm{C} 2-\mathrm{H} 2 \mathrm{~B}$ & 108.1 & $\mathrm{~N} 2-\mathrm{C} 9-\mathrm{N} 3$ & $117.56(18)$ \\
\hline $\mathrm{C} 4-\mathrm{C} 3-\mathrm{C} 2$ & $110.80(17)$ & $\mathrm{N} 2-\mathrm{C} 9-\mathrm{C} 8$ & $120.06(18)$ \\
\hline $\mathrm{C} 4-\mathrm{C} 3-\mathrm{H} 3 \mathrm{~A}$ & 109.5 & $\mathrm{~N} 3-\mathrm{C} 9-\mathrm{C} 8$ & $122.37(18)$ \\
\hline $\mathrm{C} 2-\mathrm{C} 3-\mathrm{H} 3 \mathrm{~A}$ & 109.5 & $\mathrm{C} 8-\mathrm{C} 10-\mathrm{C} 11$ & 118.05 (19) \\
\hline $\mathrm{C} 4-\mathrm{C} 3-\mathrm{H} 3 \mathrm{~B}$ & 109.5 & $\mathrm{C} 8-\mathrm{C} 10-\mathrm{H} 10$ & 121.0 \\
\hline $\mathrm{C} 2-\mathrm{C} 3-\mathrm{H} 3 \mathrm{~B}$ & 109.5 & $\mathrm{C} 11-\mathrm{C} 10-\mathrm{H} 10$ & 121.0 \\
\hline $\mathrm{H} 3 \mathrm{~A}-\mathrm{C} 3-\mathrm{H} 3 \mathrm{~B}$ & 108.1 & $\mathrm{C} 12-\mathrm{C} 11-\mathrm{C} 10$ & $119.4(2)$ \\
\hline $\mathrm{C} 3-\mathrm{C} 4-\mathrm{C} 5$ & $111.35(17)$ & $\mathrm{C} 12-\mathrm{C} 11-\mathrm{Br} 1$ & $120.17(16)$ \\
\hline $\mathrm{C} 3-\mathrm{C} 4-\mathrm{H} 4 \mathrm{~A}$ & 109.4 & $\mathrm{C} 10-\mathrm{C} 11-\mathrm{Br} 1$ & $120.42(16)$ \\
\hline $\mathrm{C} 5-\mathrm{C} 4-\mathrm{H} 4 \mathrm{~A}$ & 109.4 & $\mathrm{~N} 3-\mathrm{C} 12-\mathrm{C} 11$ & $123.84(19)$ \\
\hline
\end{tabular}




$\begin{array}{llll}\mathrm{C} 3-\mathrm{C} 4-\mathrm{H} 4 \mathrm{~B} & 109.4 & \mathrm{~N} 3-\mathrm{C} 12-\mathrm{H} 12 & 118.1 \\ \mathrm{C} 5-\mathrm{C} 4-\mathrm{H} 4 \mathrm{~B} & 109.4 & \mathrm{C} 11-\mathrm{C} 12-\mathrm{H} 12 & 118.1 \\ \mathrm{H} 4 \mathrm{~A}-\mathrm{C} 4-\mathrm{H} 4 \mathrm{~B} & 108.0 & & \\ \mathrm{C} 6-\mathrm{C} 1-\mathrm{C} 2-\mathrm{C} 3 & -56.1(2) & \mathrm{N} 1-\mathrm{C} 7-\mathrm{C} 8-\mathrm{C} 10 & -174.77(19) \\ \mathrm{C} 1-\mathrm{C} 2-\mathrm{C} 3-\mathrm{C} 4 & 56.8(2) & \mathrm{O} 1-\mathrm{C} 7-\mathrm{C} 8-\mathrm{C} 9 & -178.41(19) \\ \mathrm{C} 2-\mathrm{C} 3-\mathrm{C} 4-\mathrm{C} 5 & -55.7(2) & \mathrm{N} 1-\mathrm{C} 7-\mathrm{C} 8-\mathrm{C} 9 & 3.0(3) \\ \mathrm{C} 3-\mathrm{C} 4-\mathrm{C} 5-\mathrm{C} 6 & 54.2(2) & \mathrm{C} 6-\mathrm{N} 2-\mathrm{C} 9-\mathrm{N} 3 & 174.7(2) \\ \mathrm{C} 9-\mathrm{N} 2-\mathrm{C} 6-\mathrm{N} 1 & 4.4(3) & \mathrm{C} 6-\mathrm{N} 2-\mathrm{C} 9-\mathrm{C} 8 & -5.9(3) \\ \mathrm{C} 9-\mathrm{N} 2-\mathrm{C} 6-\mathrm{C} 5 & -116.3(2) & \mathrm{C} 12-\mathrm{N} 3-\mathrm{C} 9-\mathrm{N} 2 & 180.0(2) \\ \mathrm{C} 9-\mathrm{N} 2-\mathrm{C} 6-\mathrm{C} 1 & 124.1(2) & \mathrm{C} 12-\mathrm{N} 3-\mathrm{C} 9-\mathrm{C} 8 & 0.6(3) \\ \mathrm{C} 7-\mathrm{N} 1-\mathrm{C} 6-\mathrm{N} 2 & 1.1(3) & \mathrm{C} 10-\mathrm{C} 8-\mathrm{C} 9-\mathrm{N} 2 & 179.6(2) \\ \mathrm{C} 7-\mathrm{N} 1-\mathrm{C} 6-\mathrm{C} 5 & 120.3(2) & \mathrm{C} 7-\mathrm{C} 8-\mathrm{C} 9-\mathrm{N} 2 & 1.8(3) \\ \mathrm{C} 7-\mathrm{N} 1-\mathrm{C} 6-\mathrm{C} 1 & -118.4(2) & \mathrm{C} 10-\mathrm{C} 8-\mathrm{C} 9-\mathrm{N} 3 & -1.0(3) \\ \mathrm{C} 4-\mathrm{C} 5-\mathrm{C} 6-\mathrm{N} 2 & -170.86(16) & \mathrm{C} 7-\mathrm{C} 8-\mathrm{C} 9-\mathrm{N} 3 & -178.8(2) \\ \mathrm{C} 4-\mathrm{C} 5-\mathrm{C} 6-\mathrm{N} 1 & 69.1(2) & \mathrm{C} 9-\mathrm{C} 8-\mathrm{C} 10-\mathrm{C} 11 & 0.6(3) \\ \mathrm{C} 4-\mathrm{C} 5-\mathrm{C} 6-\mathrm{C} 1 & -51.8(2) & \mathrm{C} 7-\mathrm{C} 8-\mathrm{C} 10-\mathrm{C} 11 & 178.3(2) \\ \mathrm{C} 2-\mathrm{C} 1-\mathrm{C} 6-\mathrm{N} 2 & 171.29(17) & \mathrm{C} 8-\mathrm{C} 10-\mathrm{C} 11-\mathrm{C} 12 & 0.2(3) \\ \mathrm{C} 2-\mathrm{C} 1-\mathrm{C} 6-\mathrm{N} 1 & -68.9(2) & \mathrm{C} 8-\mathrm{C} 10-\mathrm{C} 11-\mathrm{Br} 1 & 179.67(16) \\ \mathrm{C} 2-\mathrm{C} 1-\mathrm{C} 6-\mathrm{C} 5 & 52.7(2) & \mathrm{C} 9-\mathrm{N} 3-\mathrm{C} 12-\mathrm{C} 11 & 0.3(3) \\ \mathrm{C} 6-\mathrm{N} 1-\mathrm{C} 7-\mathrm{O} 1 & 176.9(2) & \mathrm{C} 10-\mathrm{C} 11-\mathrm{C} 12-\mathrm{N} 3 & -0.6(4) \\ \mathrm{C} 6-\mathrm{N} 1-\mathrm{C} 7-\mathrm{C} 8 & -4.5(3) & \mathrm{Br} 1-\mathrm{C} 11-\mathrm{C} 12-\mathrm{N} 3 & 179.88(18) \\ \mathrm{O} 1-\mathrm{C} 7-\mathrm{C} 8-\mathrm{C} 10 & 3.8(3) & & \\ & & & \end{array}$

Hydrogen-bond geometry $\left(\AA,{ }^{\circ}\right)$

\begin{tabular}{lllll}
\hline$D-\mathrm{H} \cdots A$ & $D-\mathrm{H}$ & $\mathrm{H} \cdots A$ & $D \cdots A$ & $D-\mathrm{H} \cdots A$ \\
\hline $\mathrm{N} 1-\mathrm{H} 1 N \cdots \mathrm{N} 3^{\mathrm{i}}$ & $0.83(2)$ & $2.52(2)$ & $3.337(2)$ & $169(2)$ \\
$\mathrm{N} 2-\mathrm{H} 2 N \cdots{ }^{i i}$ & $0.83(2)$ & $1.98(2)$ & $2.807(2)$ & $175(2)$ \\
\hline
\end{tabular}

Symmetry codes: (i) $-x+1, y-1 / 2,-z+1 / 2$; (ii) $-x+1, y+1 / 2,-z+1 / 2$. 